\title{
Review Article \\ Technology Review of Modern Gas Turbine Inlet Filtration Systems
}

\author{
Melissa Wilcox, ${ }^{1}$ Rainer Kurz, ${ }^{2}$ and Klaus Brun ${ }^{1}$ \\ ${ }^{1}$ Machinery, Southwest Research Institute, San Antonio, Tx, USA \\ ${ }^{2}$ Systems Analysis, Solar Turbines Incorporated, San Diego, CA, USA \\ Correspondence should be addressed to Melissa Wilcox, melissa.wilcox@swri.org
}

Received 13 October 2011; Accepted 18 December 2011

Academic Editor: Mauro Venturini

Copyright ( 2012 Melissa Wilcox et al. This is an open access article distributed under the Creative Commons Attribution License, which permits unrestricted use, distribution, and reproduction in any medium, provided the original work is properly cited.

\begin{abstract}
An inlet air filtration system is essential for the successful operation of a gas turbine. The filtration system protects the gas turbine from harmful debris in the ambient air, which can lead to issues such as FOD, erosion, fouling, and corrosion. These issues if not addressed will result in a shorter operational life and reduced performance of the gas turbine. Modern day filtration systems are comprised of multiple filtration stages. Each stage is selected based on the local operating environment and the performance goals for the gas turbine. Selection of these systems can be a challenging task. This paper provides a review of the considerations for selecting an inlet filtration system by covering (1) the characteristics of filters and filter systems, (2) a review of the many types of filters, (3) a detailed look at the different environments where the gas turbine can operate, (4) a process for evaluating the site where the gas turbine will be or is installed, and (5) a method to compare various filter system options with life cycle cost analysis.
\end{abstract}

\section{Introduction}

Gas turbines ingest a large amount of ambient air during operation. Because of this, the quality of the air entering the turbine is a significant factor in the performance and life of the gas turbine. A filtration system is used to control the quality of the air by removing harmful contaminants that are present. The selection of the filtration system can be a daunting task, because there are many factors to consider. The system should be selected based on the operational philosophy and goals for the turbine, the contaminants present in the ambient air, and expected changes in the contaminants in the future due to temporary emission sources or seasonal changes. This paper outlines the primary considerations for selecting and installing a gas turbine inlet filtration system. First, the consequences that can occur due to improper inlet filtration are reviewed, then the different characteristics are discussed, after this the components of a filtration system and considerations for the operating environment are outlined, and lastly, a procedure for quantitatively comparing inlet filtration system options is provided.

\section{Consequences of Poor Inlet Filtration}

When the quality of the air entering the gas turbine is not well controlled, there are several consequences which can occur. Some of the most common degradation mechanisms are reviewed below including erosion, fouling, and corrosion.

2.1. Erosion. Erosion occurs when solid or liquid particles approximately $10 \mu \mathrm{m}$ and larger impact rotating or stationary surfaces in the gas turbine. The particles will impact the surface and remove tiny particles of metal which eventually lead to changes in the geometry of the surface. This change in geometry causes deviations in the air flow path, roughening of smooth surfaces, alteration of clearances, and reduction of cross-sectional areas of metal components possibly in high stressed regions. Erosion is a non-reversible process; therefore, the gas turbine components must be replaced in order to regain their original condition. However, particles $10 \mu \mathrm{m}$ and larger are easily removed by commercial filters [1-3].

2.2. Fouling. Fouling of compressor blades is an important mechanism leading to performance deterioration in gas 
turbines over time. Fouling is caused by the adherence of particles to airfoils and annulus surfaces. Particles that cause fouling are typically smaller than 2 to $10 \mu \mathrm{m}$. Smoke, oil mists, carbon, and sea salts are common examples. Fouling can be controlled by an appropriate air filtration system and often reversed to some degree by detergent washing of components. The adherence is impacted by oil or water mists. The result is a build-up of material that causes increased surface roughness and to some degree changes the shape of the airfoil (if the material build up forms thicker layers of deposits). Fouling in turn causes a decrease in the performance of the gas turbine.

Commercial filters can remove the majority of particles that cause fouling. But there are several submicron particles that are difficult to remove from the flow stream. The buildup of particles not removed by the inlet filtration system is removed with the use of compressor washing. This process recovers a larger portion of the compressor performance but cannot bring the gas turbine back to its original condition [1-5].

2.3. Corrosion. When chemically reactive particles adhere to surfaces in the gas turbine, corrosion can occur. Corrosion that occurs in the compressor section is referred to as "cold corrosion" and is due to wet deposits of salts, acid, and aggressive gases such as chlorine and sulfides. Corrosion in the combustor and turbine sections is called "hot corrosion." It is also referred to as a high temperature corrosion. Hot corrosion requires the interaction of the metal surface with another chemical substance at elevated temperatures. Hot corrosion is a form of accelerated oxidation that is produced by the chemical reaction between a component and molten salts deposited on its surface. Hot corrosion comprises a complex series of chemical reactions, making corrosion rates very difficult to predict. It is the accelerated oxidation of alloys caused by the deposit of salts (e.g., $\mathrm{Na}_{2} \mathrm{SO}_{4}$ ). Type I or high temperature hot corrosion occurs at a temperature range of 1346 to $1742^{\circ} \mathrm{F}\left(730\right.$ to $\left.950^{\circ} \mathrm{C}\right)$. Type II or low temperature hot corrosion occurs at a temperature range of 1022 to $1346^{\circ} \mathrm{F}\left(550\right.$ to $\left.730^{\circ} \mathrm{C}\right)$. Some of the more common forms of hot corrosion are sulfidation, nitridation, chlorination, carburization, and vanadium, potassium, and lead hot corrosion. Sulfidation Hot Corrosion requires the interaction of the metal surface with sodium sulfate or potassium sulfate, salts that can form in gas turbines from the reaction of sulfur oxides, water, and sodium chloride (table salt) or potassium chloride, respectively. It is usually divided into Type I and Type II hot corrosion, and Type I hot corrosion takes place above the melting temperature of sodium sulfate $\left(1623^{\circ} \mathrm{F}\left(884^{\circ} \mathrm{C}\right)\right)$, while Type II occurs below this temperature. Hot corrosion is caused by the diffusion of sulfur from the molten sodium sulfate into the metal substrate which prevents the formation of the protective oxidation film and results in rapid removal of surface metal. One should note that for hot corrosion to occur both sulfur and salt (e.g., sodium chloride or potassium chloride or chloride) have to be present in the very hot gas stream in and downstream of the combustor. Sulfur and salt can come from the inlet air, from the fuel, or water (if water is injected). The
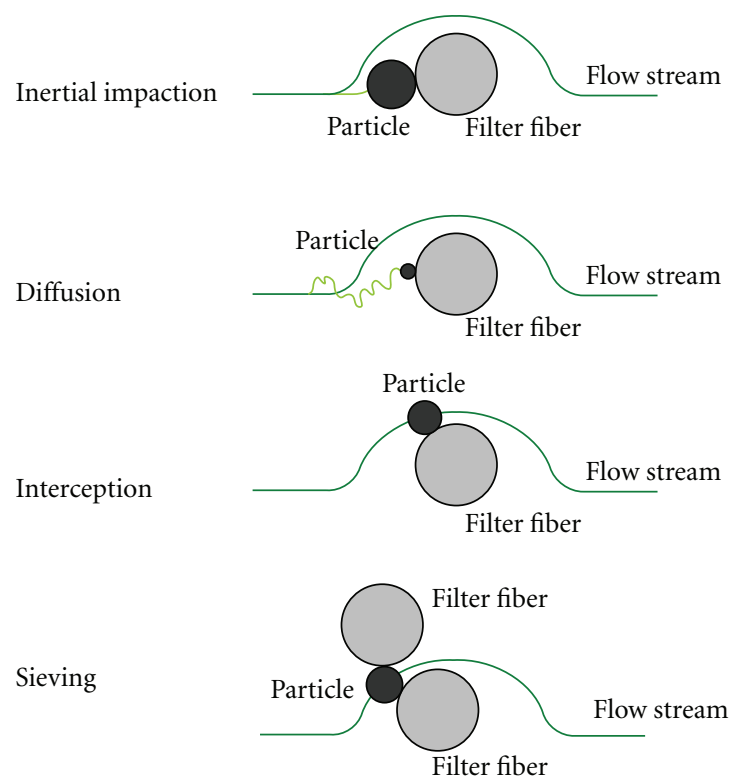

Figure 1: Common filtration mechanism [29].

potassium hot corrosion mechanism is similar to sulfidation but is less frequently observed in gas turbines, unless the fuel contains significant quantities of potassium.

Corrosion is a nonreversible degradation mechanism. Therefore, corroded components must be replaced in order to regain the original gas turbine performance. Corrosion also initiates or advances other damage mechanisms in the gas turbine. For example, corrosion can intrude into cracks or other material defects and accelerates crack propagation [1-3].

\section{Filtration Characteristics}

3.1. Filtration Mechanisms. Filters in the filtration system use many different mechanisms to remove contaminants from the air. The filter media, fiber size, packing density of the media, particle size, and electrostatic charge influence how the filter removes contaminants. Each filter typically has various different mechanisms working together to remove the contaminants. Four filtration mechanisms are shown in Figure 1.

The first filtration mechanism is inertial impaction. This type of filtration is applicable to particles larger than $1 \mu \mathrm{m}$ in diameter. The inertia of the large heavy particles in the flow stream causes the particles to continue on a straight path as the flow stream moves around a filter fiber. The particulate then impacts and is attached to the filter media and held in place as shown in the top picture of Figure 1. This type of filtration mechanism is effective in high-velocity filtration systems.

The next filtration mechanism, diffusion, is effective for very small particles typically less than $0.5 \mu \mathrm{m}$ in size. Effectiveness increases with lower flow velocities. Small particles interact with nearby particles and gas molecules. Especially in turbulent flow, the path of small particles 
When a new filter looses the charge mechanism, the efficiency drops down significantly (dashed line)

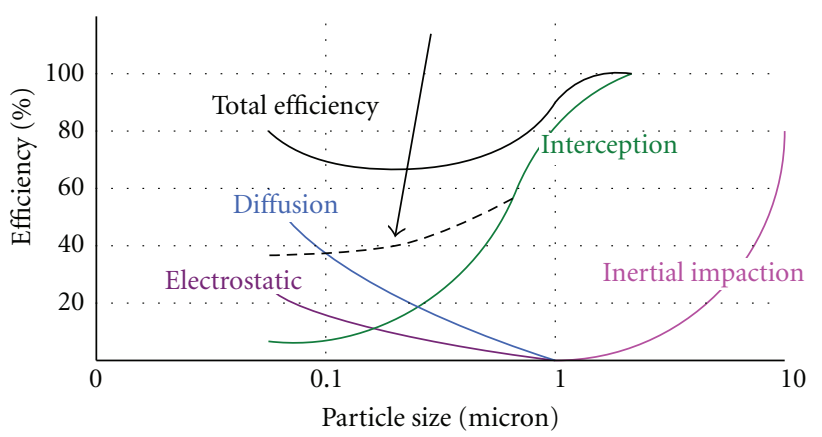

FIgURE 2: Combination of filtration mechanisms to obtain filter efficiency at various particle sizes [29].

fluctuates randomly about the main stream flow. As these particles diffuse in the flow stream, they collide with the fiber and are captured. The smaller a particle and the lower the flow rate through the filter media leads to a higher probability that the particle will be captured.

The next two filtration mechanisms are the most well known: interception and sieving. Interception occurs with medium-sized particles that are not large enough to leave the flow path due to inertia or not small enough to diffuse. The particles will follow the flow stream where they will touch a fiber in the filter media and be trapped and held. Sieving is the situation where the space between the filter fibers is smaller than the particle itself, which causes the particle to be captured and contained.

Another mechanism not shown in Figure 1 is electrostatic charge. This type of filtration is effective for particles in the 0.01 to $1 \mu \mathrm{m}$ size range (Figure 2). The filter works through the attraction of particles to a charged filter. In gas turbine applications, this charge is applied to the filter before installation as a result of the manufacturing process. Filters always lose their electrostatic charge over time because the particles captured on their surface occupy charged surface area, therefore neutralizing their electrostatic charge. As the charge is lost, the filter efficiency for small particles will decrease. On the other hand, as the filter is loaded, the filtration efficiency increases, thus counteracting the effect of lost charge to some extent. This will offset some of the loss of filtration efficiency due to the lost charge. Figure 2 shows a comparison of a filter's total efficiency based on the various filtration mechanisms that are applied. The figure shows the difference between the filter's efficiency curve before and after the charge is lost. The performance of the filter should be based on the discharged condition $[6,7]$.

3.2. Filter Efficiency and Classification. Filter efficiency is a broad term. In general, the filter efficiency is the ratio of the weight, volume, area, or number of particles captured in the filter to the weight, volume, area, or number of the particles entering the filter, respectively. A general efficiency calculation is shown in (1), where $W$ is the variable for which efficiency is being calculated. The efficiency can be expressed in several ways: maximum, minimum, or average lifetime value. Many filters have poor performance against small particles at the beginning of their lives, but as the filter media becomes loaded with particles, it is able to catch smaller particles. In this case, the average efficiency would actually be higher than the initial efficiency. Some of the filters will never reach the quoted maximum efficiency before they are replaced

$$
\eta=\frac{W_{\text {entering }}-W_{\text {leaving }}}{W_{\text {entering }}} * 100 \% .
$$

Filter efficiency is a trade-off against the pressure loss across the filter. Normally, the filtration system pressure loss will increase with an increase in filtration efficiency. As filters become more efficient, less dust penetrates through them. Also, the air flow path is more constricted with higher efficiency filters. This leads to higher pressure loss. Filter engineers must determine the acceptable pressure loss and efficiency for their application. Studies have shown that a higher pressure loss due to using a high efficiency filter has a lower effect on gas turbine power degradation than poor inlet air quality.

The efficiency of a filter cannot be stated as a general characteristic. The filter efficiencies vary with particle size, typically being lower for small particles and higher for large particles. They also vary with operational velocity. Filters designed for medium and low velocities will have a poor performance at higher velocities and vice versa. Therefore, a particle size range and flow velocity must be associated with the stated efficiency. For example, a filter may have 95 percent filtration efficiency for particles greater than $5 \mu \mathrm{m}$ at a volumetric flowrate of $3000 \mathrm{cfm}\left(5097 \mathrm{~m}^{3} / \mathrm{h}\right)$, but the efficiency could be reduced to less than 70 percent for particles less than $5 \mu \mathrm{m}$ or at a volumetric flowrate of $4000 \mathrm{cfm}\left(6796 \mathrm{~m}^{3} / \mathrm{h}\right)$.

Filters are rated for performance based on standards established in the United States of America and Europe. These filter ratings are based on the results of standard performance tests. In the United States, ASHRAE standard 52.2:2007 outlines the requirements for performance tests and the methodology to calculate the efficiencies. In this standard, the efficiencies are determined for various ranges of particles sizes. The filter is given a Minimum Efficiency Reporting Value (MERV) rating based on its performance on the particle size ranges (particle count efficiency) and the weight arrestance (weight efficiency). The weight arrestance is a comparison of the weight of the dust penetrating the filter to the dust feed into the flow stream. In this standard, a filter with a MERV of 10 will have 50 to 65 percent minimum efficiency for particles 1 to $3 \mu \mathrm{m}$ in size and greater than 85 percent for particles 3 to $10 \mu \mathrm{m}$ in size $[8,9]$.

The European standards used to determine performance are EN 779:2002 and EN 1822:2009. EN 779:2002 is used to rate coarse and fine efficiency filters. EN 1822:2009 presents a methodology for determining the performance of high efficiencies filters: Efficient Particulate Air filters (EPA), High 
TABLE 1: Summary of filter classification for ASHRAE 52.2:2007 [10], EN 779:2002 [11], and EN 1822:2009 [12-16].

\begin{tabular}{|c|c|c|c|c|c|c|c|c|}
\hline \multicolumn{5}{|c|}{ ASHRAE 52.2:2007 [10] } & \multicolumn{2}{|c|}{ EN 779:2002 [11] } & \multicolumn{2}{|c|}{ EN 1822:2009 [12-16] } \\
\hline \multirow{2}{*}{$\begin{array}{l}\text { ASHRAE } \\
\text { filter class }\end{array}$} & \multicolumn{3}{|c|}{$\begin{array}{l}\text { Average particles size efficiencies } \\
\text { in } X-Y \text { micron }(\%)\end{array}$} & \multirow{3}{*}{$\begin{array}{l}\text { EN filter } \\
\text { class }\end{array}$} & \multirow{3}{*}{$\begin{array}{l}\text { Average separation } \\
\text { efficiency }\left(A_{m}\right)\end{array}$} & \multirow{3}{*}{$\begin{array}{l}\text { Average separation } \\
\text { efficiency }\left(\mathrm{E}_{\mathrm{m}}\right)\end{array}$} & \multirow{3}{*}{$\begin{array}{l}\text { Total filtration } \\
\text { separation } \\
\text { efficiency }(\%)\end{array}$} & \multirow{3}{*}{$\begin{array}{l}\text { Local filtration } \\
\text { separation } \\
\text { efficiency }(\%)\end{array}$} \\
\hline & $\mathrm{E}_{1}$ & $\mathrm{E}_{2}$ & $\mathrm{E}_{3}$ & & & & & \\
\hline MERV & $0.3-1.0$ & $1.0-3.0$ & $3.0-10.0$ & & & & & \\
\hline 1 & & & $<20$ & G1 & $50 \leq \mathrm{A}_{\mathrm{m}}<65$ & & & \\
\hline 2 & & & $<20$ & & & & & \\
\hline 3 & & & $<20$ & $\mathrm{G} 2$ & $65 \leq \mathrm{A}_{\mathrm{m}}<80$ & & & \\
\hline 4 & & & $<20$ & & & & & \\
\hline 5 & & & $20-35$ & & & & & \\
\hline 6 & & & $35-50$ & G3 & $80 \leq \mathrm{A}_{\mathrm{m}}<90$ & & & \\
\hline 7 & & & $50-70$ & & & & & \\
\hline 8 & & & $>70$ & G4 & $90 \leq \mathrm{A}_{\mathrm{m}}$ & & & \\
\hline 9 & & $<50$ & $>85$ & & & & & \\
\hline 10 & & $50-65$ & $>85$ & F5 & & $40 \leq \mathrm{E}_{\mathrm{m}}<60$ & & \\
\hline 11 & & $65-80$ & $>85$ & & & & & \\
\hline 12 & & $>80$ & $>90$ & F6 & & $60 \leq \mathrm{E}_{\mathrm{m}}<80$ & & \\
\hline 13 & $<75$ & $>90$ & $>90$ & F7 & & $80 \leq \mathrm{E}_{\mathrm{m}}<90$ & & \\
\hline 14 & $75-85$ & $>90$ & $>90$ & F8 & & $90 \leq \mathrm{E}_{\mathrm{m}}<95$ & & \\
\hline \multirow[t]{2}{*}{15} & $85-95$ & $>90$ & $>90$ & F9 & & $95 \leq \mathrm{E}_{\mathrm{m}}$ & & \\
\hline & & & & E10 & & & 85 & \\
\hline \multirow[t]{2}{*}{16} & $>95$ & $>95$ & $>95$ & E11 & & & 95 & \\
\hline & & & & E12 & & & 99.5 & \\
\hline \multicolumn{9}{|l|}{17} \\
\hline 18 & & & & $\mathrm{H} 13$ & & & 99.95 & 99.75 \\
\hline \multicolumn{9}{|l|}{19} \\
\hline \multirow[t]{4}{*}{20} & & & & $\mathrm{H} 14$ & & & 99.995 & 99.975 \\
\hline & & & & U15 & & & 99.9995 & 99.9975 \\
\hline & & & & U16 & & & 99.99995 & 99.99975 \\
\hline & & & & U17 & & & 99.999995 & 99.9999 \\
\hline
\end{tabular}

Note: Correlations between ASHRAE and EN standard are approximate.

Efficiency Particulate Air filter (HEPA), and Ultra Low Particulate Air filter (ULPA). In EN 779:2002, the performance is found with average separation efficiency, which is an average of the removal efficiency of $0.3 \mu \mathrm{m}$ particles at four test flowrates (particle count efficiency) for fine filters and with an average arrestance (weight efficiency) for coarse particle filters. This standard rates the filters with a letter and number designation: G1 to G4 (coarse filters) and F5 to F9 (fine filters). Filter performance is determined by the Most Penetrating Particle Size efficiency (MPPS) in EN 1822:2009. The MPPS is defined as the particle size, which has the minimum filtration efficiency or maximum penetration during the filter testing. The particle sizes tested range from 0.15 to $0.3 \mu \mathrm{m}$. The filter efficiency is calculated based on particle count. These filters are given a rating of E10 to E12 for EPAtype filters, H13 to H14 for HEPA-type filters, and U15 to U17 for ULPA filters. Table 1 gives a general overview of the efficiencies for each filter rating and a comparison of the filter ratings between American and European standards [10-16].
If an engine ingests $220 \mathrm{lb} /$ year ( $100 \mathrm{~kg} /$ year) of contaminants if there were no filtration system in a typical offshore application, an F5 filter would reduce this to about $46 \mathrm{lb} /$ year (21 kg/year), an F6 filter to $13 \mathrm{lb} /$ year $(6 \mathrm{~kg} /$ year), an F7/E10 filter system to $0.44 \mathrm{lb} /$ year $(0.20 \mathrm{~kg} /$ year $)$, and an F7/F9/E10 system to as little as $0.11 \mathrm{lb} /$ year $(0.05 \mathrm{~kg} /$ year $)$. This indicates two conclusions: while large particles have an impact on fouling degradation, a significant amount is due to the finer particles. The overall contaminant ingestion can be influenced by several orders of magnitude by using an appropriate air filtration system. Also, with filtration systems of this type, there are virtually no particles larger than a few microns entering the engine [17].

3.3. Filter Pressure Loss. As mentioned above, a higher pressure loss occurs with a more efficient filter due to air flow restrictions. Pressure loss has a direct impact on the gas turbine performance, as it causes a reduction in compressor inlet pressure. For the compressor to overcome the inlet 
system losses, the gas turbine will consume more fuel, and it also has a reduced power output. As the pressure loss increases the power decreases and the heat rate increases linearly. A $0.2 \mathrm{inH} 2 \mathrm{O}(50 \mathrm{~Pa})$ reduction of pressure loss can result in a 0.1 percent improvement in power output. Typical pressure losses on inlet filtration systems can range from 2 to 6 inH2O (500 to $1500 \mathrm{~Pa}$ ) [18].

The filter's performance needs to be assessed for the full pressure loss range over its life, not just when it is new. The pressure loss will increase over the lifetime of the filter. Therefore, one can expect a lower gas turbine performance over the life of the filter, or filters have to be changed or cleaned periodically in order to maintain a low pressure loss. The change of pressure loss over time is highly dependent upon the filter selection and the type and amount of contaminants experienced.

3.4. Filter Loading (Surface or Depth). During operation as the filter collects particles, it is slowly loaded until it reaches a "full" state. This state is usually defined as the filter reaching a specified pressure loss, or when the end of maintenance interval. Filters are loaded in two different ways: surface and depth loading.

Depth loading is the type of filtration where the particles are captured inside of the filter material. To regain the original pressure loss or condition, the filter must be replaced.

The other type of filter is a surface-loaded filter. With this type of loading, the particles collect on the surface of the filter. Few of the particles may penetrate the fiber material, but not enough to call for a replacement of the filter. Surface loaded filters are most commonly used in, but not restricted to, self-cleaning systems, because the dust can easily be removed with pulses of air once the filter differential pressure reaches a certain level. Once the filter is cleaned, the pressure loss across the filter will be close to its original condition. The surface loaded filter's efficiency actually increases as the surface is loaded with dust, because a dust cake develops on the surface of the media, creating an additional filtration layer, and also decreases the amount of available flow area in the filter media $[19,20]$.

3.5. Face Velocity. Filtration systems are distinctively classified as high, medium, or low velocity systems. The velocity of the filtration system is defined as the actual volumetric air flow divided by the total filter face area. Low velocity systems have air flow at less than $500 \mathrm{ft} / \mathrm{min}(2.54 \mathrm{~m} / \mathrm{s})$ at the filter face. Medium velocities are in the range of 610 to $680 \mathrm{ft} / \mathrm{min}$ ( 3.1 to $3.45 \mathrm{~m} / \mathrm{s}$ ). High velocity systems have air flows at the filter face in excess of $780 \mathrm{ft} / \mathrm{min}(4 \mathrm{~m} / \mathrm{s})$.

3.5.1. High Velocity Systems. Historically, high velocity systems are used on marine vessels and offshore platforms where space and weight are premiums. However, today, low, medium, and high velocity systems are found on marine and offshore applications. High velocity systems have the advantages of reduced size (cross-sectional area), weight, and initial cost. Filter efficiencies for small particles are significantly lower than those of lower velocity systems, and dust holding capacities are lower.

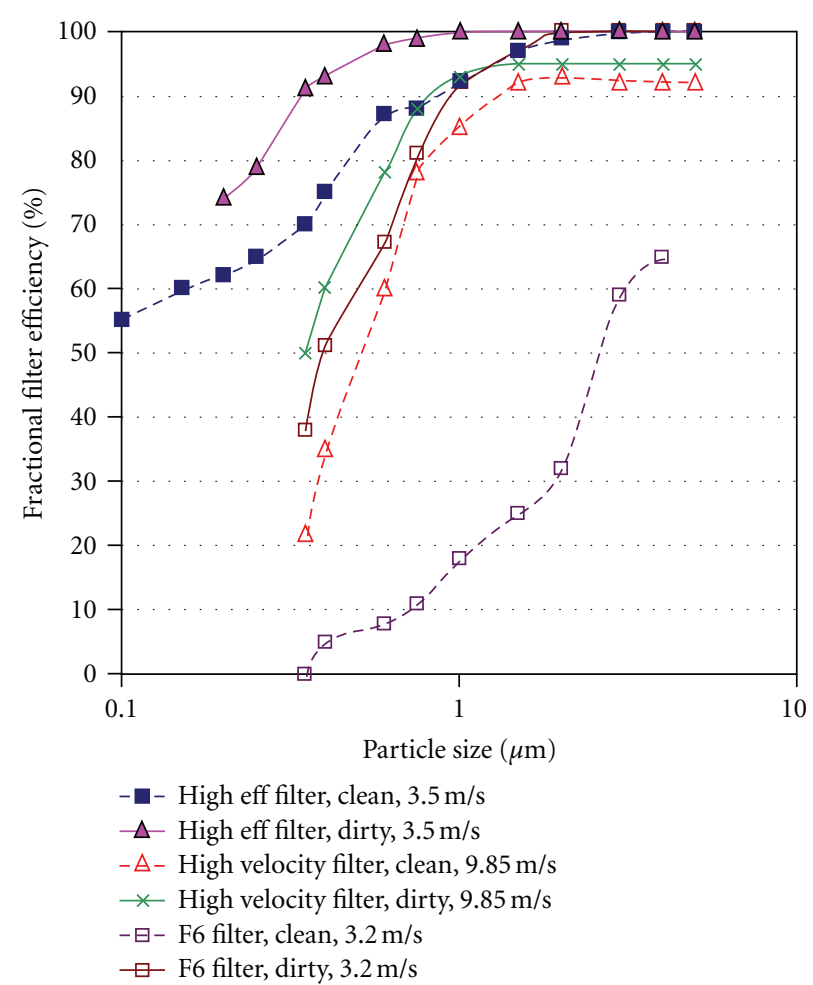

FIGURE 3: Comparison of fractional efficiency for filter elements from different suppliers and different face velocities in new and dirty conditions [25].

High velocity systems typically use vane separators upstream, and often also downstream, of the filter media to remove water from the air stream. For the vanes to work effectively, higher flow velocities are necessary. Ultimately, this type of system requires more filter replacements when compared to the lower velocity system of similar performance $[21,22]$.

3.5.2. Low Velocity Systems. Low velocity systems are the standard on land-based applications; however, high velocity systems are also used in some coastal applications. The low velocity systems are characterized by large inlet surface areas, large filter housings, and usually multiple stages of filters. The two or three stage filters provide an advantage over high velocity systems, because they have a high efficiency filter stage as the final stage to remove many small particles (especially salt) below $1 \mu \mathrm{m}$. Recently developed filter media can also keep water from penetrating the media, and thus entering the gas turbine. The lower velocity also provides a lower pressure loss or higher filtration efficiency. Using prefilters to remove the majority of the particles, the life of the high efficiency filters is extended. Overall, low velocity systems can be more effective at reducing the mass of contaminants that enter a system, thus extending the water wash intervals for the engine (Figure 3 ).

3.6. Water and Salt Effects. Many environments where gas turbines operate will have wet ambient conditions. This could be in a tropical environment where it rains a significant 
TABLE 2: Different types of moisture experience in inlet filtration systems $[2,23]$.

\begin{tabular}{lc}
\hline Description & Liquid Size $(\mu \mathrm{m})$ \\
\hline Humidity & vapor form \\
Smog (more smoke than humidity) & 0.01 to 2 \\
Cooling tower aerosols & 1 to 50 \\
Water mist & 1 to 50 \\
Clouds and fog & 2 to 150 \\
Water spray (ship wake, ocean spray) & 10 to 500 \\
Drizzle & 50 to 400 \\
Rain & 400 to 1000 \\
\hline
\end{tabular}

amount of time or coastal location with ocean or lake mist. Table 2 is a list of the different types of moisture that can be experienced together with their particle size. The difference between filter operation in wet and dry conditions can be significant. In some cases, the pressure loss across a filter can increase significantly even with a little moisture. This is true for cellulose fiber filters which swell when they are wet. These filters will also retain the moisture that can lead to long periods of time when the pressure loss across the filter is elevated.

Salt can have a direct effect on the life of a gas turbine if not removed properly. It is often carried into the engine dissolved in water spray. Salt can lead to fouling and corrosion. Gas turbine manufacturers usually recommend stringent criteria on the amount of salt which can be allowed to enter the gas turbine (less than $0.01 \mathrm{ppm}$ ). In coastal environments, the airborne salt can easily range from 0.05 to $0.5 \mathrm{ppm}$ on a typical day. If the filtration system is not equipped to handle the salt, it can enter the compressor and the hot section of the gas turbine. Salt is present in the air, either as salt dust or dissolved in seawater, and contains sodium chloride, magnesium chloride, and calcium sulfate. Salt may also come from localized sources such as a dry salt bed $[2,23]$. The salt on compressor blades must be removed through water washing methods or direct scrubbing of the blades.

\section{Components of a Filtration System}

In order to protect the gas turbine from the variety of contaminants present in the ambient air, several filtration devices are used. Each of the devices used in modern filtration systems are discussed below.

4.1. Weather Protection and Trash Screens. Weather louvers or hoods and trash screens are the most simplistic of the filtration mechanisms, but they are important in order to reduce the amount of moisture and solid contaminants, which enter the main filtration system. These are not classified as filters, but they are part of the filtration system and provide assistance in removal of large objects or contaminants carried in the flow stream.

Weather hoods are sheet metal coverings on the entrance of the filtration system (see Figure 4). The opening of

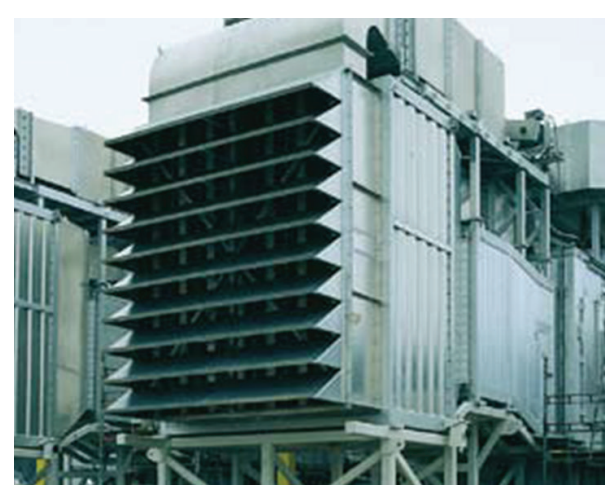

Figure 4: Weather Hood on inlet filtration system [34].

the hood is pointed downward so the ambient air must turn upwards to flow into the inlet filtration system. The turning of the air is effective at minimizing rain and snow penetration. Weather hoods and louvers are used on the majority of inlet filtration systems, and they are essential for systems in areas with large amounts of rainfall or snow. Weather hoods or another comparable weather protection system are strongly recommended for all systems with high efficiency filter.

After the weather hood is a series of turning vanes called weather louvers, which redirect the air so that it must turn. The weather louvers are also effective at minimizing water and snow penetration. After the weather hood or louver is a trash or insect screen. Trash screens capture large pieces of paper, cardboard, bags, and other objects. The screens also deflect birds, leaves, and insects. Screens that are installed specifically for preventing insects entering the filtration system are referred to as insect screens. These screens will have a finer grid than trash screens. Weather hoods, louvers, trash screens, and insect screens are used on the majority of filtration systems due to their inexpensive cost and construction, and negligible pressure loss [9].

4.2. Anti-Icing Protection. Anti-icing protection is used in climates with freezing weather. Freezing climates with rain or snow can cause icing of inlet components, which can result in physical damage to inlet ducts or to the gas turbine compressor. This ice can also affect the performance of the gas turbine. If ice forms on filter elements, then ice on those filters will be blocking the flow path, which will cause the velocity at the other filters to increase. This causes a decrease in filtration efficiency. Also, the filter elements with ice can be damaged. Figure 5 shows an example of ice formation on filters due to cooling tower drift.

Heaters, compressor bleed air, or self-cleaning filters are often used in the inlet system in frigid environments to prevent the build-up of ice on the inlet bell mouth or filter elements. It should be noted that any location in the inlet system (even past the filter system) that creates a pressure drop can potentially have ice formation. In some plant operations, the Inlet Guide Vanes (IGVs) are used for flow control at part load operations. If the IGVs are partially closed, then under the right weather conditions, ice build can 


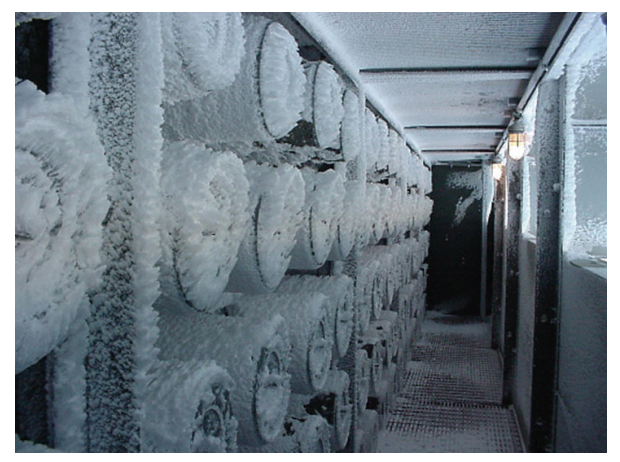

Figure 5: Pulse cartridge filters with frost build up due to cooling tower drift [29].

occur at this location. In order to prevent ice build-up in this situation, it may be necessary to limit the closer of the IGVs or have provisions to heat the inlet air to avoid ice formation.

4.3. Inertial Separators. Inertial separation takes advantage of the physical principles of momentum, gravity, centrifugal forces, and impingement, and the physical difference between phases to cause particles to be moved out of the gas stream in such a way that they can be carried off or drained. The higher momentum of the dust or water particles contained in the air stream causes them to travel forward, while the air can be diverted to side ports and exit by a different path than the dust. There are many types of inertial separators, but the ones commonly used with gas turbine inlet filtration are vane and cyclone separators $[7,9]$.

4.4. Moisture Coalescers. In environments with high concentration of liquid moisture in the air, coalescers are required in order to remove the liquid moisture. The coalescer works by catching the small water droplets in its fibers. As the particles are captured, they combine with other particles to make larger water droplets. Coalescers are designed to allow the droplets to either drain down the filter or be released back into the flow stream. If the larger drops are released, then they are captured downstream by a separator. Figure 6 shows an example of how the droplet size distribution changes across the coalescer which releases the droplets $[7,9]$.

4.5. Prefilters. The air has a mixture of large and small particles. If a one-stage high efficiency filter is used, the build-up of large and small solid particles can quickly lead to increased pressure loss and filter loading. Prefilters are used to increase the life of the downstream high efficiency filter by capturing the larger solid particles. Therefore, the high efficiency filter only has to remove the smaller particles from the air stream which increases the filter life. Prefilters normally capture solid particles greater than $10 \mu \mathrm{m}$, but some prefilters will also capture the solid particles in the 2 to $5 \mu \mathrm{m}$ size range. These filters usually consist of large diameter synthetic fiber in a disposable frame structure. Bag filters are also commonly used for prefilters. These offer higher surface area that reduces the pressure loss across the filter $[7,9]$. In

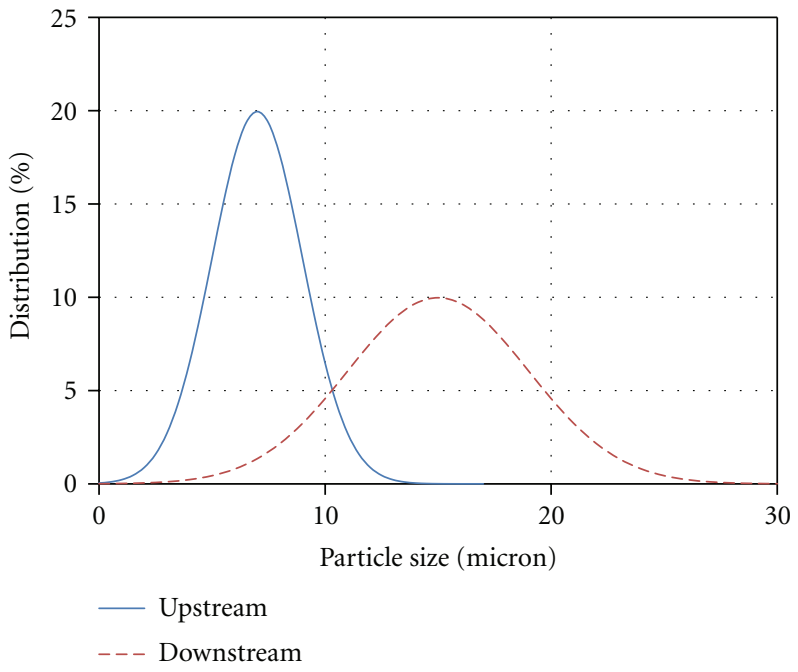

Figure 6: Coalescer droplet formation distribution [29].

many installations, the prefilters can be exchanged without having to shut the engine down.

4.6. High-Efficiency Filters. As discussed above, there are filters for removing larger solid particles, which prevent erosion and FOD. Smaller particles which lead to corrosion, fouling, and cooling passage plugging, are removed with high efficiency filters. These types of filters have average separations greater than 80 percent. Three common types of high efficiency filters are EPA, HEPA, and ULPA. EPA and HEPA filters are defined as having a minimum efficiency of 85 percent and 99.95 percent, respectively, for all particles greater than or equal to $0.3 \mu \mathrm{m}$. ULPA filters have a minimum efficiency of 99.9995 percent for particles the same size or larger than $0.12 \mu \mathrm{m}$ [11-16]. Often, these names are used loosely with discussion of high efficiency filtration. However, the majority of the high efficiency filters used in gas turbine inlet filtration do not meet these requirements.

The high-efficiency filters used with gas turbines have pleated media that increase the surface area. In order to achieve the high filtration efficiency, the flow through the filter fiber is highly restricted which creates a high pressure loss, unless the face velocity is kept low. The pleats help reduce this pressure loss. Initial pressure loss on high efficiency filters can be up to $1 \mathrm{inH} 2 \mathrm{O}(250 \mathrm{~Pa})$ with a final pressure loss in the range of $2.5 \mathrm{inH} 2 \mathrm{O}(625 \mathrm{~Pa})$ for rectangular filters and $4 \mathrm{inH} 2 \mathrm{O}(2000 \mathrm{~Pa})$ for cartridge filters. The life of the filters is highly influenced by other forms of filtration upstream. If there are stages of filtration to remove larger solid articles and liquid moisture, then these filters will have a longer life. Minimal filtration before high efficiency filters will lead to more frequent replacement or cleaning. High efficiency filters are rated under various standards. The majority of filters used in gas turbines are not classified as EPA, HEPA, or ULPA. The filters used in gas turbines are rated with ASHRAE 52.2:2007 and EN 779:2002.

There are many different constructions of high efficiency type filters: rectangular, cylindrical/cartridge, and bag filters. 

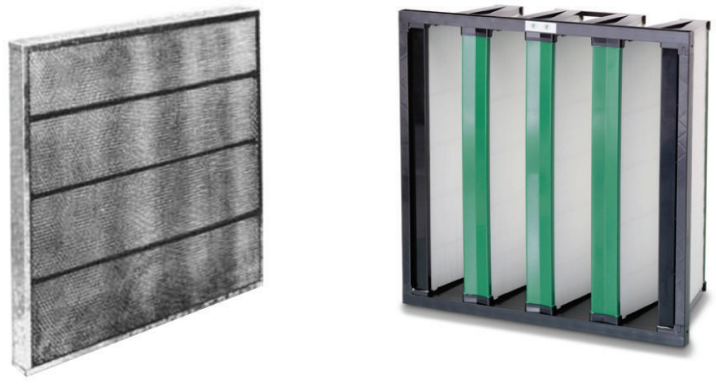

Figure 7: Rectangular high-efficiency filters $[35,36]$.

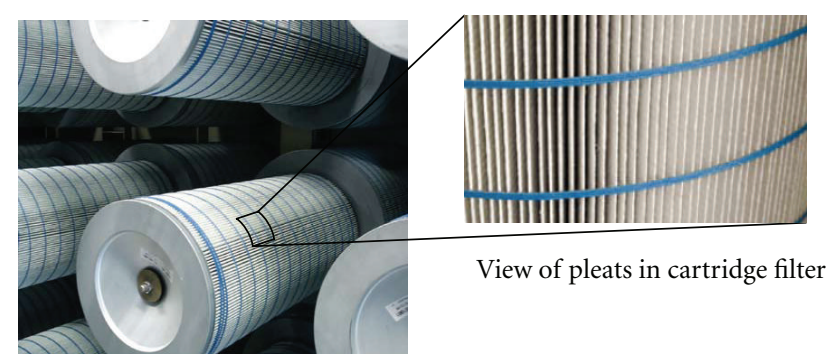

High-efficiency cartridge filters

Figure 8: High-efficiency cartridge filters [29].

The rectangular high efficiency filters are constructed by folding a continuous sheet of media into closely spaced pleats in a rectangular rigid frame. Rectangular filters are depth loaded; therefore, once they reach the maximum allowable pressure loss, they should be replaced. Two examples of rectangular high efficiency filters are shown in Figure 7. High efficiency filters can also be made from media that do not allow water to seep through the filter media.

Cartridge filters are also made up of closely spaced pleats, but they are in a circular fashion (Figure 8). Air flows radially into the cartridge. They are installed in a horizontal or vertical fashion (hanging downward). These types of filters can be depth or surface loaded. The surface loaded filters are commonly used with a self-cleaning system, but not all of them are designed for self-cleaning. Cartridge filters used in self-cleaning systems require a more robust structural design in order to protect the filter fiber media during the reverse air pulses. The more common structural support is a wire cage around the pleated media on the inside and outside of the filter. The filters shown in Figure 8 are not designed for a self-cleaning system since there are no structural supports on the outside of the filter. Self-cleaning filtration systems are discussed in the next section $[7,9]$.

4.7. Self-Cleaning Filters. All of the filters with fiber-type media previously discussed are required to be replaced once they reach the end of their usable life. In some environments, the amount of contaminants can be excessive to the point where the filters previously discussed would have to be replaced frequently to meet the filtration demand. A prime example of one of these environments is a desert with sand

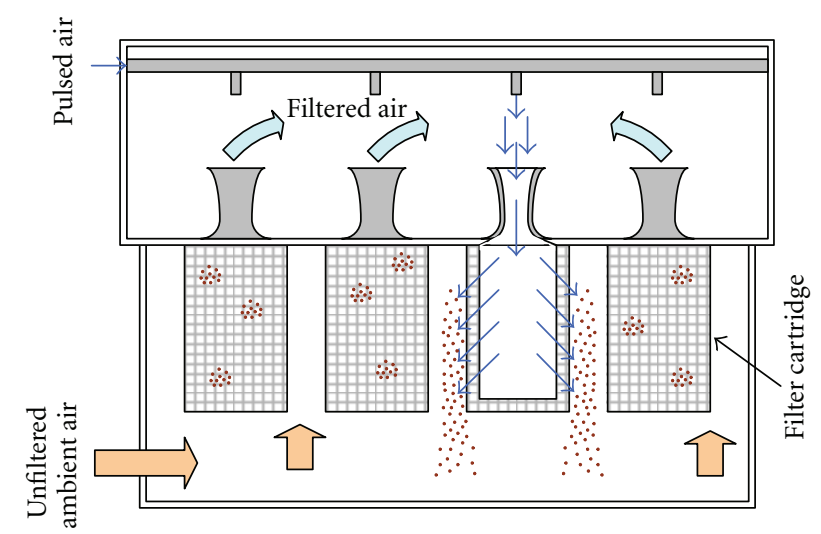

FIGURE 9: Example of operation of an updraft self-cleaning filters [29].

storms. In the 1970s, the self-cleaning filtration system was developed for the Middle East where gas turbines are subject to frequent sand storms. Since then, this system has been continually developed and utilized for gas turbine inlet air filtration.

The self-cleaning system operates primarily with surfaceloaded high-efficiency cartridge filters. The surface loading allows for easy removal of the dust, which has accumulated with reverse pulses of air (Figure 9). The pressure loss across each filter is continuously monitored. Once the pressure loss reaches a certain level, the filter is cleaned with air pulses. The pressure of the air pulses ranges from 80 to 100 psig (5.5 to 6.9 barg). The reverse jet of compressed air (or pulse) occurs for a length of time between 100 and $200 \mathrm{~ms}$. To avoid disturbing the flow and to limit the need for compressed air, the system typically only pulses 10 percent of the elements at a given time. With this type of cleaning, the filter can be brought back to near the original condition $[19,24]$.

4.8. Staged Filtration. Any gas turbine application typically needs more than one type of filter, and there are no "universal filters" that will serve all needs. Therefore, two-stage or three-stage filtration systems are used. In these designs, a prefilter or weather louver can be used first to remove erosive contaminants, rain, and snow. The second may be a lowto-medium-performance filter selected for the type of finersized particles present or a coalescer to remove liquids. The third filter is usually a high-performance filter to remove smaller particles less than $2 \mu \mathrm{m}$ in size from the air. Figure 10 shows a generalized view of a filtration arrangement. This arrangement is not correct for all cases due to the fact that the filter stages are highly influenced by the environment they are operating in.

\section{Operating Environment}

The selection of the inlet filtration system should be primarily dependent on the environment where it operates. This includes the contaminants in the ambient air from surrounding vegetation, weather events, local emissions, temporary emissions, and seasonal changes. Several different 


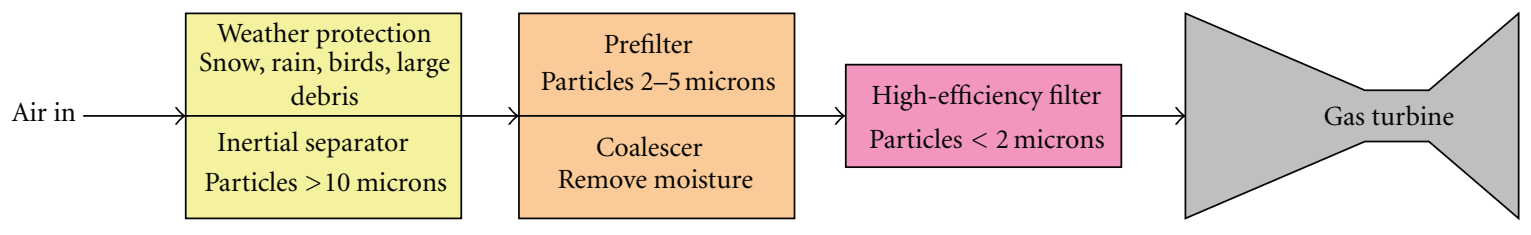

FIgURE 10: Multistage filtration system.

common environments with their typical contaminants are reviewed below.

5.1. Coastal, Marine, or Offshore. Gas turbines operating near or on the ocean are classified as being in a coastal, marine, or offshore environment. The gas turbine is in a coastal environment when the gas turbine is installed on land and within 10 miles $(16 \mathrm{~km})$ of the ocean. At distances from approximately 8 to 12 miles ( 13 to $19 \mathrm{~km}$ ) from the shoreline, the salt concentration in the air drops to natural background levels for an environment far away from the ocean [23]. The offshore and marine environments are defined as being in the middle of the ocean. The gas turbine is considered in an offshore environment when it is located at least $100 \mathrm{ft}$ $(30.5 \mathrm{~m})$ off of the ocean surface. Gas turbines located below $100 \mathrm{ft}(30.5 \mathrm{~m})$ are considered to be in a marine environment.

The primary contaminant that is a concern in the coastal, marine, and offshore environments is salt. Salt as previously discussed can lead to fouling and corrosion. Salt is prevalent in these environments due to the sea water. In coastal environments, it is present as dry contaminants (areas with lower humidity), sticky contaminants (humidity between 40 and 70 percent), or as liquid aerosols (humidity greater than 70 percent) [2]. In the offshore environment, salt is usually present in the sticky particle or liquid state. The marine environment (closest to the ocean surface) has salt present in the liquid aerosol state. In all these environments, the amount of salt depends on the wind speed and direction and the elevation of the gas turbine.

Coastal environments also have land-based contaminants that must be removed from the air. These will be discussed in more detail below. Offshore environments have industrial contaminants such as exhaust fumes, by-products of maintenance (such as dust from grit blasting), and unburned hydrocarbons from flares. Many of these particles are on the submicron size; therefore, high efficiency filtration is often employed. The marine environment most often does not have as many additional contaminants to remove from the air. However, when a ship is near a coast, land-based contaminants may be present. In addition, icing in colder environments is often a concern. Icing can also be an issue in offshore and coastal environments.

The filtration system for a coastal environment is similar to that of a land-based environment, which will be discussed later. However, in coastal environment it is important to have mist eliminators for water and salt water removal and high efficiency filtration for salt removal. The filtration system in an offshore environment is similar to a coastal filtration system but may have increased air velocity due to size and weight limitations. The filtration system on a marine vessel is most commonly comprised of a vane-coalescer-vane system. This system has two vane axial separators with a coalescer in between them. This system is a high velocity system that is designed for removing salt water. It has limited solid particle removal capability $[2,23,25,26]$.

5.2. Land-Based Environment. The land-based environment is very diverse. It can be classified in many different ways depending on weather patterns, vegetation, and local emission sources. Several land-based environments are described below.

5.2.1. Desert. The desert is classified as an area with a dry and hot climate. Large amount of dust is present and there is little vegetation. Sand storms are common and can quickly load filters to their maximum dust holding capacity. The main regions of the world which can be characterized by desert like environments are across the Sahara desert in Africa, the Middle East, and parts of Asia. However, small localized areas with high dust concentrations do exist. These can include gas turbines installed near quarries, dried lakebeds, loess, industrial areas, dirt tracks, dry agricultural land, and construction sites. There are three typical conditions that exist in the desert: clean air, dust haze, and sand storms. Dust is the main contaminant in the desert for these conditions. This can be sand or other fined grained material such as desert pavement. Desert pavement is the layer of large stones left on the floor of the desert. While these stones are not harmful in their solid state, they can easily be broken by human or animal traffic and crumbled into fine particles. These particles can range from large $(500 \mu \mathrm{m})$ to very fine (submicron size). Due to the lack of vegetation and protection of the ground dust from the wind, more dust can be lofted into the air than in other environments. This leads to a high concentration of dust.

The filtration systems in deserts are usually solely designed for dust removal. However, some desert locations experience periods of dense fog and high humidity. This is especially true for deserts near a coastal region. The moisture can collect on the surface of cartridge filters on self-cleaning systems and cause the dirt to form a cake on the filter. This cake of dust can significantly reduce the effectiveness of filtration and pulse cleaning. If fog and high humidity are present at the desert-type site, then this should be considered for the filtration system.

Dust loads in the desert can range from mild (low wind) to fairly high (dust storms). Conventional non-selfcleaning filtration systems can quickly become loaded and 
require frequent filter change outs. Also, high pressure losses can trigger a shutdown if they become excessive. In order to avoid the constant maintenance and labor required for changing filters out, a self-cleaning system is needed. Filtration systems without self-cleaning filters have proven to be more expensive due to the labor cost and maintenance required with filter replacements $[26,27]$.

5.2.2. Arctic. The arctic environment is characterized by freezing weather (below $32^{\circ} \mathrm{F}\left(0^{\circ} \mathrm{C}\right)$ ) for an extended period of time. The location will not necessarily be classified as arctic for the entire year. It will have other land-based contaminants, which must be considered. However, the arctic seasons of the year will influence the design of the inlet filtration system.

Ice build-up is the primary concern in this environment during the cold months. Ice can form from the ingestion of snow or freezing rain and also due to the depression or cool humid air in the inlet system. Placement of the inlet of the filtration system, weather hoods with large openings (referred to as snow hoods), and self-cleaning filters is often adequate to protect against the ingestion of snow and freezing rain. To prevent the formation of ice from the depression of cool humid air requires an anti-icing system such as recirculated exhaust air or a compressor bleed.

In addition to ice, warm season contaminants must be considered for the design of the inlet filtration system. These contaminants can be similar to any of the other land-based environments discussed in this section [26].

5.2.3. Tropical. Tropical areas are characterized by hot climate, high humidity, monsoons, high winds, and insect swarms. Due to the extensive vegetation, there is not much erosion concern. It is considered a low-dust environment. The area has little seasonal variation with the exceptions of periods of intense rainfall. Typhoons, dust, insects, and the remoteness of systems in the tropics should be considered when choosing the correct system.

The main contaminants in this area are water (from rain), insects, and salt (if the location is near a shoreline). Dust is minimal, since the overgrown vegetation protects the ground dust from winds. Of course, there are always exceptions to this. If the gas turbine is installed in a construction site, then the dust levels will be higher than normal. Also, unpaved roads can contribute to the dust in the environment. Pollen can be an issue. Salt will be present in aerosol form due to the high humidity and moisture present.

The filtration systems for tropical environments are specifically built to handle large amounts of rain. Weather hoods are used as a primary defense. Extended area insect screens are used for blocking insects. These screens have a lower air velocity (in the range of $260 \mathrm{ft} / \mathrm{min}(1.3 \mathrm{~m} / \mathrm{s})$ ), which allows the insects to move away from the screens. This prevents obstruction of the inlet air flow. This is followed by a mix of prefilters, coalescers, and vane separators. The water removal system must be designed in order to handle the highest expected water ingestion and prevent corrosion. If this is not done, then water will be able to travel farther downstream in the inlet filtration system. Any prefilters or high efficiency filters used should be selected to prevent water travel through the filter. If water is allowed to penetrate the filter, then it can absorb the capture soluble contaminants and transport them through the filter into the gas turbine. This can have detrimental effects if salt is being removed from the air stream. These filters should also be selected for the expected contaminants such as pollen and road dust $[26,28]$.

5.2.4. Rural. The rural countryside is a diverse environment. Depending upon where the gas turbine is located in this environment, it can be subjected to hot, dry climate, rain, snow, and fog throughout the year. The majority of the year there is a nonerosive environment with low dust concentrations in the range of 0.02 to $0.1 \mathrm{ppm}$ ( 0.01 to 0.05 grains per $1000 \mathrm{ft}^{3}\left(28.3 \mathrm{~m}^{3}\right)$ ). The area can be near a local forest or be near agricultural activities.

The contaminants in this environment vary depending on the season. Throughout the year, insects and airborne particulate will need to be filtered. If the gas turbine is installed near an agricultural area, then during plowing and harvesting season, the concentration of dust will increase. During plowing, insecticides and fertilizers will be airborne. At harvest, the particles or grains from cutting plants down will be lofted into the air. The particles that travel to the gas turbine are relatively small (less than $10 \mu \mathrm{m}$ ), unless strong winds are present to carry large particles. Gas turbines near forests may not have as high dust concentration. The foliage of the forest will protect the ground dust from being lifted by the wind. With the change in season, snow, rain, fog, pollen, airborne seeds, and insects will be present. This climate has one of the most diverse filtration requirements as compared to other environments.

These systems are typically comprised of three stages: weather hood, prefilter, and high-efficiency filter. The weather hood protects the filters farther downstream from rain and snow. They also minimize the amount of dust entering the filtration system. Insect screens are used, especially if insects are present in swarms during parts of the year. The prefilter is used to remove any erosive dust present in the air. The prefilter also protects the high efficiency filter from being overloaded too quickly. The high efficiency filter removes the smaller particles. If the gas turbine is installed near an agricultural area, the filter engineer may consider a self-cleaning system. This type of system would be beneficial during plowing or harvest season when the air has a high erosive dust concentration. A self-cleaning system can also be beneficial in an area with a dry, cold climate during the winter season. It can effectively prevent ice from forming on the filter elements and influencing the gas turbine operation [26].

5.2.5. Large City. Large cities can experience all the types of gas turbine degradation: corrosion, erosion, and fouling. Contaminants from many different sources ensure the requirement of a multistaged filtration system.

All different types of weather can occur throughout the year in a large city. The amount of contaminants varies throughout the season as discussed above for the rural 
countryside. One example is salt or grit that is laid down on icy roads during the winter. The city also has smog and pollution. These can also be seen in the countryside due to high winds, but are much more concentrated in the city. Some other considerations for large cities are noise issues and vandals.

The system has a multistage approach with specific filters installed for the local contaminants. Weather hoods are used the majority of the time due to the changing weather conditions with seasons. This protects the system from rain, snow, and windy conditions. The filtration system is composed of a prefilter and a high-efficiency filter. The prefilter removes the larger erosive particles. The highefficiency filter is typically of the non-self-cleaning type with rectangular filters or cartridges filters. The self-cleaning systems are not used due to the sticky aerosols present in the air. If freezing conditions are expected, then an anti-icing system is included. Urban/industrial areas typically do not have airborne particulate concentrations that warrant the use of self-cleaning filtration systems, but self-cleaning systems are used successfully in these areas, when these are in regions of heavy snow and minimal sticky contaminants [26].

5.2.6. Industrial Area. Many gas turbines are installed in heavy industrial areas. These locations can be in any of the environments discussed above, but they have additional concerns. There are several emission sources in an industrial location, which contribute to the contaminants that must filtered out.

The most prevalent contaminant in industrial areas is contaminants from exhaust stacks. These can be in the form of particles, gases, and aerosols. Many of the particles emitted by the exhaust stack are in the submicron size range. These size particles are difficult to filter and can collect on compressor blades and cause fouling. The gases emitted in the exhaust can contain corrosive chemicals. For example, exhaust gases from fossil fuel plants has $\mathrm{SO}_{x}$, which contains sulfur. Sulfur is one of the corrosive components that can lead to hot corrosion in the turbine section. Gas cannot be removed by mechanical filtration. Aerosols also present a challenge. These are typically on the submicron size and difficult to filter. Many of these aerosols are sticky, and when they are not removed by the filters, they stick to compressor blades, nozzles, and other surfaces. One example of this already mentioned in this guideline is the compressor blade fouling due to oil vapors.

Industrial locations can also experience contaminants that are not typically seen, unless near a localized source. Some examples of these are dust from mining operations, sawmills, foundries, and other industrial facilities. Also, if the gas turbine is near a petrochemical plant, the air may be contaminated with specific chemicals. These chemicals could be harmless, but they also could have corrosive properties.

One commonality between all industrial locations is that the inlet of the filtration system is subjected to the local plant emissions. This condition typically requires a more robust high-efficiency filtration system to remove fine particles that are entrained in the air. One way to reduce the amount of emissions that are ingested into the inlet is to direct the inlet air flow away from these emission sources. Several recommendations in regards to the inlet placement and site layout are discussed in a later section. Even so, there are still some emissions that are ingested by the turbine. Additional filter elements should be included in the filtration system to address these emission particles. For example, if the industrial location is near an open coal storage site, then the gas turbine should have prefilters and high-efficiency filters to remove the coal dust that is in the air.

One contaminant that is often in the air at industrial locations is sticky aerosols. These aerosols can be from oil vapors from lubrication systems or unburned hydrocarbons emitted from exhaust stacks. These aerosols are very difficult to remove from the air and often lead to blade fouling. Highefficiency filters should be used to minimize the aerosol's effect on the gas turbine, but a compressor washing scheme is needed to keep the compressor blades clean and to minimize the effects of fouling on gas turbine performance [26].

5.3. Temporary and Seasonal Contaminant Sources. In many of the applications discussed above, temporary or seasonal conditions are mentioned. As gas turbines become more advanced and more sensitive to the inlet air quality, it becomes more important to address these conditions.

In order to address seasonal changes, the expected conditions must first be defined. During the design phase, the air quality at the site where the gas turbine is going to be installed should be monitored for at least 1 year. This will provide the filter engineer with information about which contaminants they can expect in each season. Also, the filter engineer should map out any potential construction, agricultural, or dust-generating projects that will occur in the first 5 to 10 years of the life of the gas turbine. Combining the expected contaminants will allow the filter engineer to develop a more holistic approach to their inlet filtration.

Currently, the majority of the filtration systems installed have a fixed filtration system. The number of stages, types of filters, and level of filtration remain constant throughout the operation. If the future site for the gas turbine is expected to have high variability in the type of contaminants experienced (temporary or seasonal), the filter engineer may consider a filtration system which allows the use of many different filters. This would then allow the filtration system to be adapted to the current conditions.

5.4. Site Layout. The layout of the site where the gas turbine is installed can have a significant effect on the type and amount of contaminants that need to be removed from the inlet air. This has been mentioned in several of the environmental-type discussions above but is summarized here for completeness. Listed below are general recommendations. Gas turbine manufacturers may have their own set of guidelines for placing the gas turbine [29].

(i) When installing other combustion-type equipment, such as a diesel engine, near the gas turbine, the exhaust of the equipment should be directed away from the gas turbine inlet. This reduces the possibility of the exhaust gas entering the gas turbine inlet 
system. This exhaust can contain unburned hydrocarbons or corrosive gases.

(ii) Cooling towers can be a major source of aerosol drift. Cooling towers are open to the atmosphere and, therefore, release aerosols into the air due to agitation from cross winds and the flow of the water down the tower. The water in the cooling tower also contains water treatment chemicals that could be detrimental to the gas turbine. The drift of aerosols from a cooling tower is confined within a few hundred feet. If possible, the gas turbine inlet should be positioned away from cooling towers and placed upstream of the prevailing wind direction to minimize the aerosol drift. CFD can be a useful tool to model how the wind will carry aerosols over to the gas turbine inlet. This will help the filter engineer to properly place the gas turbine to minimize cooling tower drift effects.

(iii) Pressure relief valves are installed on many gas lines and equipment to protect the equipment in case of an over pressurization event. The vents to these relief devices should be directed away from the gas turbine inlet. Release of any hydrocarbon could result in high concentration ingestion at the filtration system. The filters at the inlet to the gas turbine do not remove gas phase contaminants.

(iv) Piping connections on gas, fluid, or steam lines will generally leak after some time. The leaks at these connections can impact the filtration system. Piping should be routed away from the inlet in order to prevent this influence.

(v) Lube oil vents should be directed away from the inlet to prevent oil vapor ingestion.

(vi) The exhaust of the gas turbine should be directed away from the inlet of the gas turbine. Carbon smoke and hydrocarbon fumes released at the exhaust could lead to accelerated fouling of the compressor blades.

(vii) The gas turbine inlet system should not be directed toward or installed near any exhaust stacks. These exhaust stacks release chemical exhaust and unburned hydrocarbons, which can lead to compressor fouling and corrosion.

(viii) Avoid placing the inlet near gravel or dirt roads. The dust thrown into the air from vehicle traffic and wind can be carried into the inlet of the gas turbine.

(a) If the gas turbine is operated during construction activities, consider adding more robust filters to remove the excess dirt that will be ingested.

(ix) Direct the inlet away from any open storage of coal, salt, or other grainy particles. The wind can carry the smaller grains from the storage area into the inlet of the gas turbine.
5.5. Site Evaluation. As discussed previously, there are several different types of environments where a gas turbine can operate. Also, there are many possible local, seasonal, and temporary contaminants that can be present. Therefore, each gas turbine installation site has a unique make-up of contaminants. When selecting the inlet filtration system, this make-up should be determined. This includes determining what contaminants and how much are present at the site. Once this information is known, the types of filters needed and filtration efficiency required can be established. Below is a list of items that should be considered when evaluating the site where the gas turbine will be installed $[9,29]$ :

(i) environment where the gas turbine will be installed: Coastal, marine, offshore, desert, arctic, tropical, industrial area, rural countryside, or large city,

(ii) contaminants present in that environment,

(iii) local contaminants (mining operating, foundries, agricultural activities, inland salt lakes, etc.)

(iv) temporary contaminants (construction activity, dirt roads, etc.),

(v) future emission sources (new industrial facility or residential development),

(vi) site layout (vents and exhaust, cooling tower drift, open storage of grainy particles, etc.),

(vii) weather patterns.

\section{Life Cycle Cost Analysis}

When selecting a filtration system, the filter engineer is burdened with deciding the level of quality they want their system to achieve. This includes the efficiency of filtration, the particle size to be filtered, the amount of maintenance that will be needed to maintain the filtration system, what rate of degradation is acceptable for the gas turbine, the required availability and reliability of the gas turbine, what type of washing scheme will be used (online, offline, or a combination of both), and cost of the filtration system. The cost impact of each of the items mentioned can be quantified. A Life Cycle Cost (LCC) analysis provides a convenient means to compare different filtration system options quantitatively.

6.1. Life Cycle Cost Basics. This section covers the inputs that should be considered for the LCC analysis for a filtration system. It also provides methods to calculate the cost impact for each input. This type of analysis focuses on the overall or lifetime cost of a system. It is a tool that estimates the total cost to purchase, install, operate, maintain, and dispose of equipment. This analysis can assist in determining the best design options, which will minimize the overall cost of a system.

It is important to include initial cost in the analysis, but it is just as important to include operation and maintenances cost. The operating and maintenance cost over the life of a piece of equipment can have a more significant effect, especially if a poorly designed system is chosen. An LCC 
analysis can help to determine which system configuration can minimize lifetime costs. Some of the costs that are typically considered are shown below. Examples of how this would apply to filtration systems are provided in parentheses:

(i) initial cost (filters, filtration system, spares filters, instrumentation),

(ii) installing and commissioning costs (labor, cost of installation equipment (such as cranes), shipping costs),

(iii) energy costs (pulse system for self-cleaning filters),

(iv) operating costs (labor, inspections),

(v) maintenance (replacing filters, repairing system, labor for maintenance),

(vi) downtime (replace filters, complete offline water washes, anything outside of normal shutdowns for other maintenance),

(vii) gas turbine effects (degradation, performance loss),

(viii) decommissioning and disposal (disposal of filters).

In an LCC analysis, estimates are provided for each cost component of the system. An inflation rate can be applied to the costs which will occur later in the life of a system (such as 10 years from the installed date). Once these costs are established, they are brought back to present value using (2). The Net Present Value (NPV) term represents the value of the cost in present terms. $A$ is the value of the cost in the year it occurs. The term $i$ is the discount rate and $n$ is the year the cost occurs in. If there is a price increase (inflation) or decrease, then this can be accounted for by using (3). The term $e$ is the increase or decrease in price:

$$
\begin{gathered}
\mathrm{NPV}=A(1+i)^{-n}, \\
\mathrm{NPV}=A(1+(i-e))^{-n} .
\end{gathered}
$$

Projected costs over the lifetime of the system cannot be combined directly when calculating the LCC, because the funds spent at different times have different values to the investor. The discount rate, $i$, is used to bring the costs to present terms, where they can be directly added together, and is defined as the rate of return that is used to compare expenditures at different points in times. For example, the investor would be equally satisfied to have one amount received earlier and the other amount received later.

If a cost occurs yearly, the NPV of the total recurring costs can be calculated with (4). If inflation or price escalation is considered in the analysis, the NPV of the total recurring cost can be calculated with (5):

$$
\begin{gathered}
\mathrm{NPV}=\frac{A}{i}\left(1-[1+i]^{-n}\right), \\
\mathrm{NPV}=A\left(\frac{1+e}{1-e}\right)\left(1-\left[\frac{1+e}{1+i}\right]^{n}\right) .
\end{gathered}
$$

The NPVs must be determined for each cost. Then the cost will be added together to obtain the total NPV or LCC cost [30].
6.2. Considerations for an Inlet Filtration System. In an LCC analysis for a gas turbine inlet filtration, there are six main parameters: purchase price/initial cost, maintenance cost, availability/reliability of the gas turbine, gas turbine degradation and compressor washing, pressure loss, and failures of the filtration system or gas turbine due to inlet air quality [29].

6.2.1. Purchase Price/Initial Cost. The purchase price occurs in the first year of the LCC analysis. It is the cost to purchase and install the inlet filtration system. An estimate for this value can be obtained from the filter vendor or gas turbine manufacturer.

6.2.2. Maintenance Cost. The maintenance cost includes the cost of filter replacement and disposal and any maintenance to auxiliary systems for the inlet filtration system. It is a recurring cost that should be included in each year that the cost is acquired. This cost can be calculated based on estimated filter change out frequencies, cost of filters from vendors, labor cost for maintenance, and cost of downtime to replace filters.

6.2.3. Availability/Reliability of Gas Turbine. The availability/reliability of a gas turbine impacts the cost due to the lost production as a result of the nonavailability of the gas turbine. Filter exchanges requiring the shutdown of the engine, as well as on-crank water washing negatively, impacts the availability of the engine. On the other hand, if the engine is not used 100 percent of the time, for example, because it is a standby or peaking unit, the cost of degradation has to be adjusted accordingly.

6.2.4. Gas Turbine Degradation and Compressor Washing. Gas turbine degradation is perhaps the most important cost in the analysis. This is often the cost which drives the analysis to favor one filtration system option over another. The cost of gas turbine degradation is calculated based on the reduced power output and increased heat rate due to inlet air quality. The rate of degradation due to inlet air quality is difficult to calculate and is best found from past operating history. There are several degradation models discussed in the literature, which can provide estimates of the expected degradation rate. A few examples are the models presented by Zaba and Lombardi [31], Kurz and Brun [32], and Meher-Homji et al. [33].

Once the degradation rate is calculated the lost profit due to reduced gas turbine output can be calculated. If the gas turbine is operating at full load, then it is expected that the fuel cost will decrease due to the lower power output. For part load operations, it is expected that the fuel cost will go up since the engine will be operated at the desired power output. The change in fuel cost should be calculated based on the change in heat rate and operational philosophy and be included in the analysis. This cost should be included in each year of the analysis.

Compressor washing is often performed in a gas turbine in order to minimize the effects fouling on the performance 
of the gas turbine. The use of compressor washing may reduce the rate of degradation in the gas turbine. However, the most effective type of washing is on-crank washing, which requires that the engine is shut down. This results in a lower availability of the engine, and, associated with this, may cause the cost of lost production.

6.2.5. Pressure Loss. The pressure loss across the inlet filtration system can also have a significant effect on the cost of the inlet filtration system. An increase in the pressure loss across the filtration system leads to reduced power output from the gas turbine and an increased heat rate. The cost of these effects should be included yearly in the LCC analysis.

6.2.6. Failure/Event Cost. The last cost is any cost associated with a failure or event that occurs due to the inlet filtration system or inlet air quality. This could be a failure of a filter material, which requires shutdown for replacement or a failure of a gas turbine blade which occurred due to corrosion from poor inlet air quality. These costs are often included based on past experience with the gas turbine or other filtration systems.

\section{Conclusions}

In summary, the selection and operation of an inlet filtration system is highly dependent on the environment where the gas turbine is operating. The contaminant present in the ambient air will dictate the type filters that are used. It is important to quantify what type and size of contaminants are present in order to correctly select the filters to be used. Temporary and seasonal variations must also be considered for the inlet filtration system. A life cycle cost analysis provides a convenient method to quantify and compare various filtration system options such that the optimal system can be selected.

\section{Nomenclature}

$\begin{array}{ll}e: & \text { Escalation rate } \\ i: & \text { Discount rate } \\ n: & \text { Year cost occurs } \\ A: & \text { Cost in present value } \\ \text { EPA: } & \text { Efficient particulate air filter } \\ \text { HEPA: } & \text { High efficiency particulate air filter } \\ \text { IGV: } & \text { Inlet guide vanes } \\ \text { MERV: } & \text { Minimum efficiency reporting value } \\ \text { MPPS: } & \text { Most penetrating particle size } \\ \text { NPV: } & \text { Net present value } \\ \text { ULPA: } & \text { Ultra low particulate air filter } \\ W: & \text { Weight, volume, area, or particle number } \\ \eta: & \text { Efficiency. }\end{array}$

\section{References}

[1] R. Kurz and K. Brun, "Gas turbine tutorial-maintenance and operating practices effects on degradation and life," in Proceedings of the 36th Turbomachinery Symposium, 2007.
[2] P. T. McGuigan, "Salt in the marine environment and the creation of a standard input for gas turbine air intake filtration systems," in Proceedings of the ASME Turbo Expo Power for Land, Sea, and Air, Vienna, Austria, 2004, GT2004-53113.

[3] S. Howes, "Selecting gas-turbine inlet air systems for new, retrofit applications," Combined Cycle Journal, Second Quarter, 2004.

[4] E. Syverud, O. Brekke, and L. E. Bakken, "Axial compressor deterioration caused by saltwater ingestion," Journal of Turbomachinery, vol. 129, no. 1, pp. 119-126, 2007.

[5] T. Z. Baden, "Losses in gas turbines due to deposits on the blading," Brown Boveri Review, vol. 67, no. 12, pp. 715-722, 1980.

[6] HEPA Filtration Facts, Donaldson Filtration Solutions, 2009.

[7] Principles of Air Filtration, Mueller Environmental Design, 2009.

[8] M. Owens, Engineering Bulletin-Compressor Fouling Benhmark, AAF International, 2009.

[9] R. L. Loud and A. A. Slaterpryce, "Gas Turbine Inlet Air Treatment," GE Power Generation, GER-3419A, 1991.

[10] ASHRAE 52.2, Method of Testing General Ventilation AirCleaning Devices for Removal Efficiency by Particle Size, American Society of Heating, Refrigeration and Air-Conditioning Engineers, 2007.

[11] DIN EN 779, Particulate Air Filters for General VentilationDetermination of the Filtration Performance, European Committee for Standardization, 2002.

[12] DIN EN-1 1822, High Efficiency Air Filters_Part 1: Classification, Performance Testing, Marking, European Committee for Standardization, 2009.

[13] DIN EN-2 1822, High Efficiency Air Filters_Part 2: Aerosol Production, Measuring Equipment, Particle Counting Statistics, European Committee for Standardization, 2009.

[14] DIN EN-3 1822, High Efficiency Air Filters-Part 3: Testing Flat Sheet Filter Media, European Committee for Standardization, 2009.

[15] DIN EN-4 1822, High Efficiency Air Filters-Part 4: Determining Leakage of Filter Element (Scan Method), European Committee for Standardization, 2009.

[16] DIN EN-5 1822, High Efficiency Air Filters-Part 5: Determining The Efficiency of Filter Element, European Committee for Standardization, 2009.

[17] R. Kurz and K. Brun, "Fouling mechanisms in axial compressors," in Proceedings of the ASME Turbo Expo, Power for Land, Sea, and Air, Vancouver, Canada, 2011, GT2011-45012.

[18] Gas Turbine World 2009 GTW Handbook, vol. 27, 2009.

[19] T. J. Retka and G. S. Wylie, "Field experience with pulsejet self-cleaning air filtration on gas turbines in an arctic environment," Journal of Engineering for Gas Turbines and Power, vol. 109, no. 1, pp. 79-84, 1987.

[20] A. Klink and T. Schroth, "New solutions for improved intake air filtration of gas turbines and turbocompressors," in Proceedings of the ASME Turbo Expo Power for Land, Sea, and Air, Birmingham, UK, 1996.

[21] R. K. Mudge and S. D. Hiner, "Gas turbine intake systemshigh velocity filtration for marine gas turbine installation," in Proceedings of the ASME Turbo Expo, Power for Land, Sea, and Air, New Orleans, La, USA, 2001, 2001-GT-0584.

[22] A. D. Oswald and S. D. Hiner, "More efficient applications for naval gas turbines-addressing the mismatch between available technology and the requirements of modern naval gas turbine inlets," in Proceedings of the ASME Turbo Expo Power for Land, Sea and Air, Barcelona, Spain, 2006, GT200690305. 
[23] J. P. Stalder and J. Sire, "Salt percolation through gas turbine air filtration systems and its contribution to total contaminant level," in Proceedings of the International Joint Power Generation Conference, pp. 445-456, New Orleans, La, USA, June 2001, JPGC2001/PWR-19148.

[24] R. G. Neaman and A. W. Anderson, "Development and operating experience of automatic pulse-jet self-cleaning air filters for combustion gas turbines," in Proceedings of the Gas Turbine Conference and Products Show, New Orleans, La, USA, 1980, 80-GT-83.

[25] O. Brekke, L. Bakken, and E. Syverud, "Filtration of gas turbine intake air in offshore installations: the gap between test standards and actual operating conditions," in Proceedings of the ASME Turbo Expo, Power for Land, Sea, and Air, Orlando, Fla, USA, 2009, GT2009-59202.

[26] D. G. Hill, "Gas turbine intake systems in unusual environments," in Proceedings of the Gas Turbine Conference and Products Show, Washington, DC, USA, 1973, 73-GT-38.

[27] C. Brake, "Identifying areas prone to dusty winds for gas turbine inlet specification," in Proceedings of the Turbo Expo, Power for Land, Sea, and Air, Montreal, Canada, 2007, GT2007-27820.

[28] R. E. Cleaver, "Gas turbine filtration in tropical environments," in Proceedings of the Turbomachinery Maintenance Congress, 1990.

[29] M. Wilcox, R. Baldwin, A. Garcia-Hernandez, and K. Brun, "Guideline for gas turbine inlet air filtration systems," in Proceedings of the Gas Machinery Research Council, 2010.

[30] Europump and Hydraulic Institute, Pump Life Cycle Costs: A Guide to LCC Analysis for Pumping Systems, 1st edition, 2001.

[31] T. Zaba and P. J. Lombardi, "Experience in the operation of air filters in gas turbine installation," Brown Boveri Review, vol. 72, no. 4, pp. 165-171, 1985.

[32] R. Kurz and K. Brun, "Degradation in gas turbine systems," Journal of Engineering for Gas Turbines and Power, vol. 123, no. 1, pp. 70-77, 2001.

[33] C. B. Meher-Homji, M. Chaker, and A. F. Bromley, "The fouling of axial flow compressors-causes, effects, susceptibility and sensitivity," in Proceedings of the ASME Turbo ExpoPower for Land, Sea, and Air, Orlando, Fla, USA, 2009, GT200959239.

[34] Offshore Filter Systems, Camfil Farr Brochure, 2009.

[35] Cam GT for Turbomachinery, Camfil Farr Product Sheet, 2009.

[36] Industrial Filtration, Burgess-Manning Bulletin BM-2-301A, 2009. 

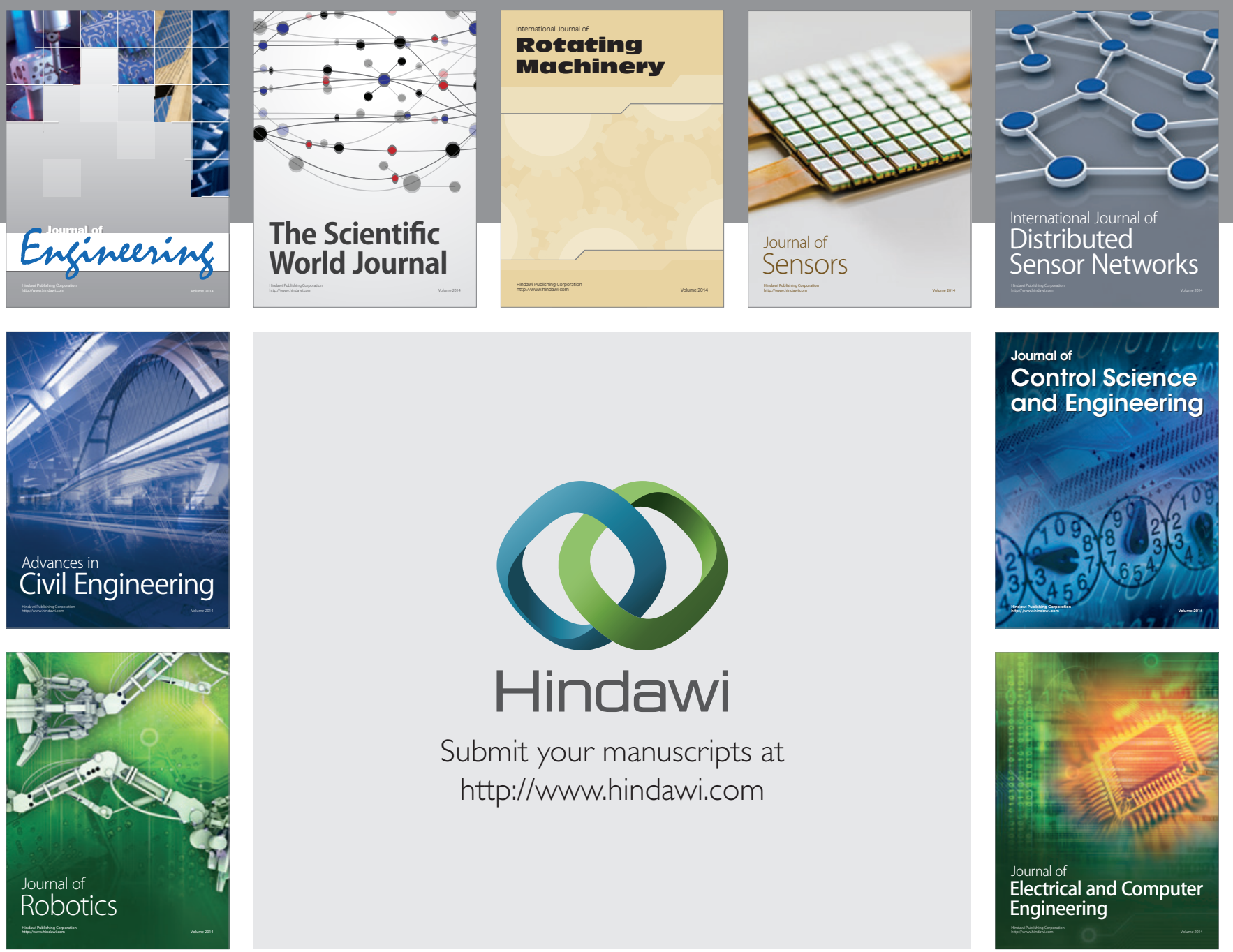

Submit your manuscripts at

http://www.hindawi.com
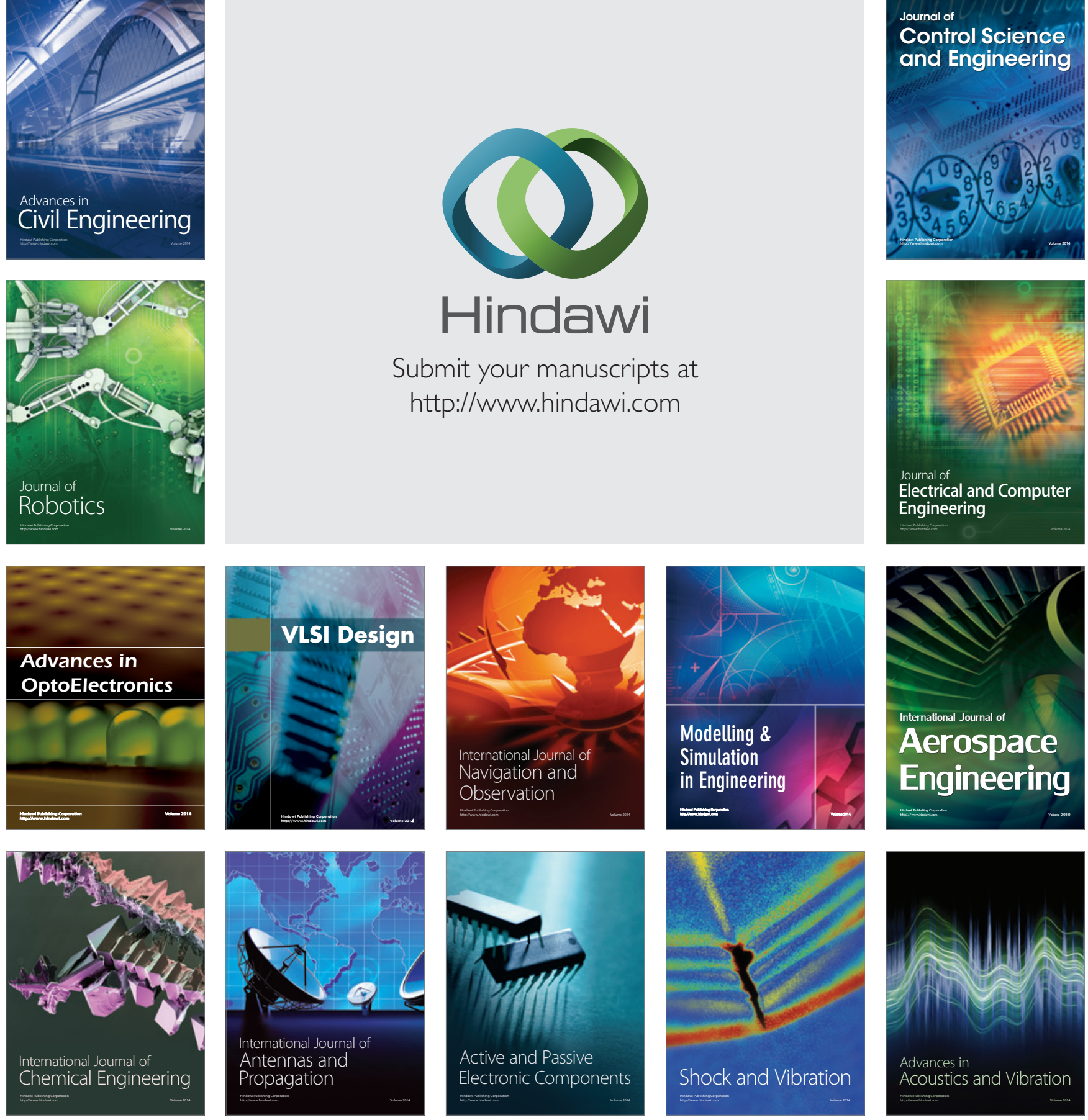